\title{
Blastoid variant of mantle cell lymphoma of the female urethra mimicking a caruncle: A rare but highly aggressive subtype case with literature review
}

\author{
Franco Palmisano ${ }^{1,2}$, Vito Lorusso ${ }^{1,2}$, Matteo Giulio Spinelli ${ }^{1}$, Paolo Guido Dell'Orto ${ }^{1}$, \\ Emanuele Montanari ${ }^{1,2}$ \\ ${ }^{1}$ Fondazione IRCCS Ca' Granda, Ospedale Maggiore Policlinico, Department of Urology, Milan, Italy; \\ ${ }^{2}$ University of Milan, Milan, Italy.
}

\begin{abstract}
Summary Primary urethral lymphoma is a rare entity without a standardized treatment protocol. We report a case of an elderly woman presenting with a caruncle associated with vaginal spotting and intermittent dysuria. She underwent surgical excision of the lesion. Histological analysis revealed a blastoid variant of mantle cell lymphoma, a previously unreported subtype. The patient received chlorambucil assisting a rapid local disease progression. She died of disseminated disease 6 months after diagnosis. A review of the lymphomas of the urethra is included.
\end{abstract}

KEY WORDS: Caruncle; Lymphoma; Urethra; Genitourinary; Mantle cell lymphoma; Blastic variant.

Submitted 10 September 2018; Accepted 27 September 2018

\section{INTRODUCTION}

Non-Hodgkin's genitourinary lymphoma as a primary extra nodal invasion is a rare condition, with few reported cases affecting the urethra (1). The most common subtypes described are mucosa-associated lymphoid tissue-type (MALT) lymphoma and diffuse large B-cell lymphoma. Herein, we report the first documented case of a blastoid variant of mantle cell lymphoma resembling a caruncle. Furthermore, to shed light on clinical features of primary urethral lymphoma, we reviewed previously reported cases.

\section{Case report}

A 84-year-old woman presented with a recent onset, fastgrowing genital nodule associated with vaginal spotting and intermittent dysuria. Her medical history was noncontributory, lacking fever, weight loss or any other systemic manifestations. There were no enlarged lymph nodes or organomegaly upon physical examination. Pelvic examination revealed a urethral caruncle arising from the posterior wall of the urethral meatus (Figure la, b). It was characterized by an erythematous appearance, firm at palpation, measuring approximately $4 \mathrm{~cm}$ at its maximum diameter. The patient underwent cystourethroscopy with a $21 \mathrm{~F}$ cystoscope revealing no bladder involvement, and surgical excision of the urethral caruncle. Histologic exam- ination revealed a urethral mucosa characterized by squamous metaplasia, subjugated by a widespread growth pattern of lymphoid proliferation, consisting of mediumsized, blastic-shaped, B-cell phenotype elements. Immunohistochemically, these cells were positive for CD20, CD5, Cyclin D1 and BcL-2, negative for CD23, CD10, BCL6, MUM1, CD3, CD30, TdT and cytokeratin. The Ki-67 index was approximately 90\%.

A diagnosis of a primary blastoid variant of mantle cell urethral lymphoma was made.

A chest, abdomen and pelvis contrast enhanced computed tomography (CT) was performed for the staging workup and revealed non-mediastinal lymphadenopathy, no focal lung lesions, a 2.5-mm angioma located at the right lobe of the liver, normal spleen, pancreas and kidneys, and no abdominal or pelvic lymphadenophaty. 18F-fluorodeoxyglucose positron emission tomography (FDG-PET) showed abnormal FDG uptake present in the external genitalia and vagina.

The patient was then referred to the Oncohematology Department and underwent two cycles of chlorambucil, despite this a rapid local disease progression was seen (Figire 1c). The patient died of disseminated disease six months after diagnosis.

\section{Discussion}

Caruncles commonly affect the postmenopausal female urethra, appearing as erythematous nodules at the posterior lip of the urethral meatus. This lesion is considered to be neither neoplastic nor preneoplastic, being composed of chronic inflammatory cells, dilated vessels and hyperplastic epithelium. In light of this, an incorrect diagnosis of lymphoma or sarcoma may occasionally be made based on the presence of bizarre stromal cells or lymphoid infiltrate (2). On the other hand, $2.3 \%$ of the caruncles were found to be malignant tumors upon histopathological analysis, usually a carcinoma or a Bowen's disease (2). In this context, a caruncle-like primary lymphoma is exceptionally rare. Since 1949, only 29 patients with urethral lymphoma have been reported (1-2), including the present case, with an age distribution ranging from 31 to 90 years old. Of these, a female 


\section{Figure 1.}

A, B. Polypoid, caruncle-like, flesh-colored lesion (Images taken during cystoscopy).

C. Clinical presentation showing disease progression 3 months after diagnosis.

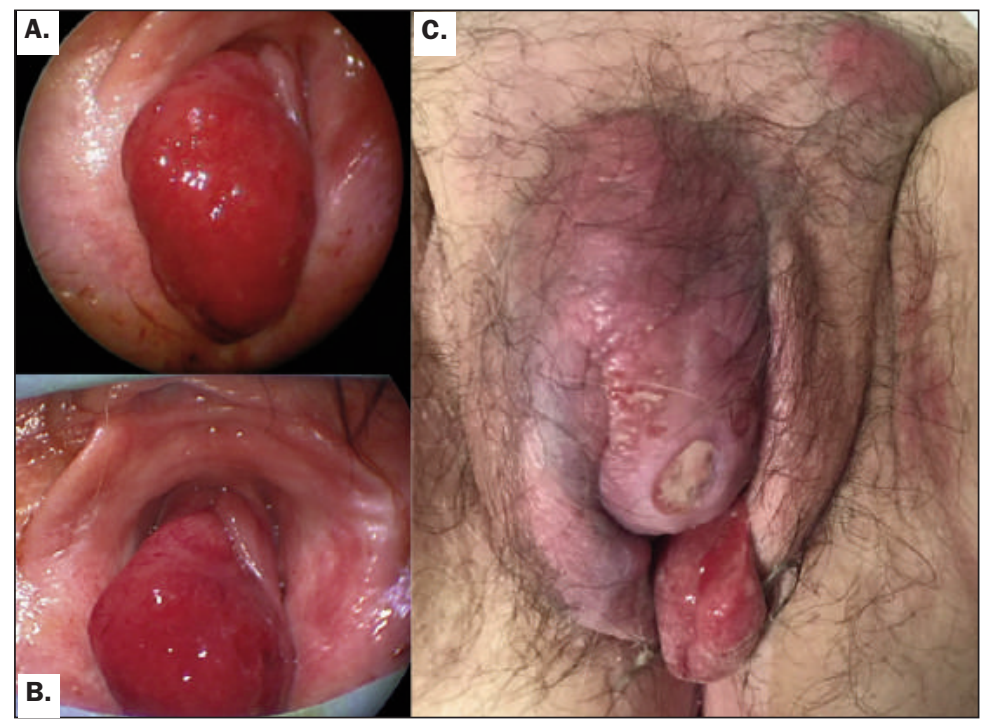

The overall mortality rate is $66 \%$ with a 14.5 month median survival time. Reported frontline therapy includes CHOP-like regimen or chlorambucil.

The blastic variant of mantle cell lymphoma is usually diagnosed upon initial presentation and more rarely in the course of common forms. Patients are predominantly male, over 60 years of age, and one third have B symptoms.

The majority of patients have an Ann Arbor stage IV disease (85\%), lymphadenopathy (82\%) and extranodal involvement (66\%), especially in the lung and pleural cavities, more rarely with a gastro-intestinal infiltration. Neither Ann Arbor staging nor bone marrow or blood involvement have been found to influence clinical outcome and there is currently no standard therapy for this disease.

\section{Conclusions}

In conclusion, the correct recognition of a primary lymphoma of the urethra is of clinical importance and should be considered in

predominance has been shown, with $75.9 \%$ of cases affecting woman. When the female urethra is involved, a caruncle like aspect is the most common presentation, having been reported in 9 cases. Other associated symptoms include spotting, hematuria, dysuria and vulvar pruritis. Cancer-specific mortality is usually relegated to a disseminate disease at diagnosis, whereas the overall 4year survival rate of caruncle-like tumors is $55.5 \%$.

Each of the previously reported 29 cases involved nonHodgkin's lymphoma, with the most common subtypes being mucosa-associated lymphoid tissue-type lymphoma and diffuse large B-cell lymphoma.

Treatments include excision, radiotherapy and chemotherapy. To our knowledge, this is the first blastoid variant of mantle cell caruncle-like lymphoma to be reported. According to Bernard et al. (3), the blastic variant form of mantle cell lymphoma is considered to be a very aggressive subtype of non-Hodgkin's lymphoma with $46 \%$ of patients who do not respond to treatment dying quickly due to disseminated disease. the differential diagnosis of a caruncle. Being the extranodal lymphoid infiltrate a diagnostic dilemma, it is of utmost importance that the pathologist has expertise concerning these tumors. An universally accepted treatment scheme is needed, and for subtypes of lymphoma with a less favorable prognosis an aggressive treatment strategy is strongly recommended.

\section{REFERENCES}

1. Al Zahrani A, Abdelsalam M, Al Fiaar A, et al. Diffuse large Bcell lymphoma transformed from mucosa-associated lymphoid tissue lymphoma arising in a female urethra treated with rituximab for the first time. Case Rep Oncol. 2012; 5:238-245.

2. Chen YR, Hung LY, Chang KC. Mucosa-associated lymphoid tissue-type lymphoma presenting as a urethral caruncle with urinary bladder involvement. Int J Urol. 2014; 21:1073-4.

3. Bernard M, Gressin R, Lefrère F, et al. Blastic variant of mantle cell lymphoma: a rare but highly aggressive subtype. Leukemia. 2001; 15:1785-91.

\section{Correspondence}

Franco Palmisano, MD (Corresponding Author)

franco.palmisano@hotmail.it

Vito Lorusso, MD

Matteo Giulio Spinelli, MD

Paolo Guido Dell'Orto, MD

Emanuele Montanari, MD

Department of Urology, IRCCS Ca' Granda Ospedale Maggiore Policlinico via della Commenda 15, 20122 Milan, Italy 